\title{
The Use of Transcranial Direct Current Stimulation (tDCS) To Reduce Impulsivity and Aggression in Adults With Intellectual Developmental Disabilities: The tDCS-RIADD Randomized Controlled Trial Protocol
}

\section{Najat Khalifa ( $\nabla$ nrk2@queensu.ca )}

Queen's University https://orcid.org/0000-0002-2690-9736

\section{Emily R Hawken}

Queen's University

\section{Andrew Bickle}

Queen's University

Jessica Jones

Queen's University

Muhammad Ayub

University College London

\section{Research Article}

Keywords: tDCS, aggression, impulsivity, developmental disability, neurostimulation

Posted Date: October 27th, 2021

DOI: https://doi.org/10.21203/rs.3.rs-617152/v1

License: (a) This work is licensed under a Creative Commons Attribution 4.0 International License.

Read Full License 


\section{Abstract \\ Background}

Challenging behaviours, in particular aggressive behaviours, are prevalent among people with intellectual developmental disabilities. Predictors of challenging behaviours are numerous, including past history of aggression, poor coping skills and impulsivity. Factors like motor or Rapid Response Impulsivity (RRI), have neurobiological underpinnings that may be amenable to change via neuromodulation using noninvasive brain stimulation techniques like transcranial Direct Current Stimulation (tDCS).

\section{Methods}

This study aims to determine the efficacy of anodal tDCS in reducing RRI and incidents of aggression in people with intellectual developmental disabilities (IDD) in residential or hospital settings. Using a single blind, randomized, sham-controlled trial design, adults with IDD, with a history of impulsivity leading to aggression, will be randomised to receive either repetitive anodal or sham tDCS applied to the left dorsolateral prefrontal cortex. Outcome measures assessing impulsivity and aggression, will be collected for up to one month following the last tDCS session.

\section{Discussion}

The results of this study may pave the way for developing targeted interventions for impulsivity and aggressive behaviours in people with IDD.

\section{Introduction}

\section{Background and rationale $\{6 a\}$}

Challenging behaviours are common among people with intellectual developmental disabilities (IDD), encompassing behaviours such as aggression, sexually inappropriate behaviours, self-injury, and criminality [1]. Research studies reported an overall prevalence rate of $10-15 \%$ for any type of challenging behaviour [2] and rates ranging from $6.1 \%$ in community settings to $40 \%$ in long-stay hospitals with significant adverse consequences, e.g., in terms of quality of life, legal consequences, breakdown of placements, and harm to others [3].

The aetiology of aggressive behaviour is multi-factorial, including physical complaints (e.g., pain, constipation, infection), behavioural phenotypes (e.g., Prader Willi syndrome), psychiatric disorders (e.g., psychosis, autism spectrum disorder), and psychosocial factors like trauma [3]. Predictors of inpatient aggression among people with IDD are numerous, including past history of aggression, poor coping skills 
and impulsivity [4, 5]. Some factors, like impulsivity, have defined neurobiological underpinnings amenable to change through biopsychosocial interventions.

A multi-dimensional construct with cognitive, motor, and temporal dimensions [6, 7], impulsivity reflects a tendency to act without thinking through the consequences of one's actions. Motor or Rapid Response Impulsivity (RRI) is a form of impulsivity that reflects failure to refrain from action initiation or to stop an ongoing or prepotent action [8]. RRI underpins several psychiatric disorders including borderline personality disorder, attention deficit hyperactivity disorder, and substance use disorders. Due to its association with criminality, poor concordance with treatment and suicidality, impulsivity is regarded as an important consideration in risk assessment and management $[6,9,10,11,12]$. Although numerous biopsychosocial approaches have been proposed to manage aggression and impulsivity in people with $\operatorname{IDD}[3,13]$, the evidence base for their efficacy is limited, highlighting the need to develop specific or adjunctive interventions.

Transcranial Direct Current Stimulation (tDCS) has been used to modulate impulsivity with some promising results $[7,14,15]$, offering great potential as a treatment for impulsive and aggressive behaviours in people with IDD. It can be used to modulate a brain network involving the left Dorsolateral Prefrontal Cortex (DLPFC) and limbic system (anterior insula, amygdala, anterior cingulate cortex). Disruption to this network, with reduced 'top-down' control from the DLPFC and an overactive limbic system results in greater impulsivity [12]. Iwabuchi et al [16] modulated this network using intermittent Theta Burst Stimulation (iTBS) applied to left DLPFC, whereby iTBS significantly dampened frontoinsular effective connectivity. This mechanism might underpin the therapeutic effects of tDCS in reducing impulsivity.

\section{Objectives $\{7\}$}

This study is the first randomized controlled clinical trial that aims to assess the efficacy of anodal tDCS in reducing RRI and incidents of aggression in people with IDD. We hypothesise that anodal tDCS applied to the left DLPFC will result in greater reductions in impulsivity and incidents of aggression than sham tDCS, demonstrating a clear treatment effect of tDCS in persons with IDD. Here, we outline the experimental protocol used to address our hypotheses.

\section{Trial design \{8\}}

A single blind, parallel arms, randomised controlled trial design will be employed in this study.

\section{Methods}

\section{Participants, interventions and outcomes}

\section{Study setting $\{9\}$}

The study involves adults with IDD residing in the community, inpatient units or care homes in the Southern Ontario Region of Canada. 


\section{Eligibility criteria $\{10\}$}

Eligible adults aged between 18 and 65, with a history IDD and aggression, will be identified using a tDCS safety questionnaire. Individuals with a history epilepsy, significant head injury or other neurological conditions, current substance misuse and those with metal or cochlear implants will be excluded. Table 1 provides more information about the inclusion and exclusion criteria.

\section{Who will take informed consent? $\{26 \mathrm{a}\}$}

The study will recruit participants from intellectual developmental disability services in the Kingston area (or Southern Ontario Region). Posters will be placed publicly at Ongwanda Research Centre in Kingston, and community living spaces were people with IDD reside. Participants will be identified by their psychiatrist, caregiver or support worker. Individuals designated to identify potential participants will ask prospective participants or their Substitute Decision Maker to complete a hospital "consent to be contacted for research" form. This form will be given to study personnel who will then contact the individual either in person at the health service, by phone or email. Study personnel (a research assistant or study coordinator) will then approach individuals that have already consented to be contacted for research purposes. If an individual is unable to provide consent, their Substitute Decision Makers will be approached. Study personnel will ensure that potential participants have not opted-out or withdrawn their consent or provided consent to be contacted for research purposes before initiating contact with participants or their Substitute Decision Makers.

Designated study personnel will review or read over the consent with the participant or their Substitute Decision Maker and answer any questions they have. Individuals must be able to understand that participation is completely voluntary and they can withdraw consent at any time and without giving a reason. To minimize coercion in the consenting process, the participant letter of information has been adapted to a grade 3 reading/comprehension level.

New information will be shared with participants and their Substitute Decision Makers at any time during their participation in the study as soon as it becomes available. Participants will be re-consented if necessary during the study at any study visit and the study will not proceed until the participant is reconsented. Participants or their Substitute Decision Makers can withdraw their consent at any time by expressing their desire to do so to study personnel.

\section{Additional consent provisions for collection and use of participant data and biological specimens $\{26 \mathrm{~b}\}$}

Not applicable.

\section{Interventions}

Explanation for the choice of comparators $\{6 b\}$ 
The comparator is sham tDCS stimulation. The fundamental principle of a sham stimulation is that participants struggle to unblind themselves to the treatment. Ten seconds of stimulation is sufficient to keep individuals blinded to their treatment arm but is not enough stimulation to modulate brain activity. The validity of this sham protocol has been published previously demonstrated [17].

\section{Intervention description $\{11 \mathrm{a}\}$}

The Soterix tDCS kit will deliver 20 minutes stimulation sessions using two $5 \times 5 \mathrm{~cm}$ sponge electrodes. The stimulation montage will comprise left DLPFC anodal or sham stimulation. The anodal electrode will be placed over the area corresponding to the left DLPFC (F5 of the EEG10-20 international system) and the reference (cathodal) electrode over the right supraorbital ridge. The active stimulation condition will use a constant current of $2 \mathrm{~mA}$, delivered via current ramps over 10 seconds at the onset and offset of stimulation, respectively. For sham stimulation, the current will be delivered only in the first 10 seconds, after which the stimulation will cease but with the electrodes still in place throughout the session.

\section{Criteria for discontinuing or modifying allocated interventions $\{11 \mathrm{~b}\}$}

Discontinuation rules include withdrawal of consent, development of a coincidental health problem/illness, exacerbation of aggressive behaviors expressed as a report of a serious violent incident and/or an increased incidence of self-harm or self-injurious behavior. Additionally, the study could stop early if the intervention significantly exacerbates aggressive, impulsive or self-harming behaviors.

\section{Strategies to improve adherence to interventions $\{11 \mathrm{c}\}$}

tDCS stimulation will be administered by the research personnel as per the study's protocol.

\section{Relevant concomitant care permitted or prohibited during the trial $\{11 \mathrm{~d}\}$}

Not applicable.

\section{Provisions for post-trial care $\{30\}$}

Patients will be informed what arm (treatment or sham) they were allocated to at the end of the study. They will also be advised to contact the study principal investigator should they experience any adverse effects following each session. Additionally, participants will continue to receive usual care at the discretion of the treating psychiatrist or care team. It usually involves a combination of behavioural approaches, psychosocial interventions and psychotropic medications.

\section{Outcomes $\{12\}$}

\section{Primary Outcome Measures:}

\section{Aggression:}


The Modified Overt Aggression Scale (MOAS) [18] will be used to measure aggressive behaviours at baseline, one week (Day 10), and one month (Day 38) following the last tDCS treatment. MOAS has four domains (Verbal Aggression, Aggression Against Property, Autoaggression and Physical Aggression) which are weighted and each rated on a 5-point scale.

\section{Impulsivity:}

The Stop Signal Task (SST) (Psytoolkit.org), a behavioural measure of RRI, will be administered at baseline and following the third tDCS treatment (Day 3) with Stop Signal Reaction Time (SSRT) as the primary outcome measure. SSRT is defined as the mean reaction time on go trials minus the mean Stop Signal Delay at which the participant successfully withholds a response on $50 \%$ of the trials. Lower SSRT values corresponds to higher impulsivity.

\section{Secondary Outcome Measures:}

\section{Maladaptive behaviours:}

The Behavior Problems Inventory Short Form (BPI) [19] is an informant-rated instrument used to assess maladaptive behaviours in people with IDD. Changes in maladaptive behaviours will be measured at baseline, one week (Day 10), and one month (Day 38) following the last tDCS treatment.

\section{Trait impulsivity:}

To account for the effects of trait impulsivity on outcome measures, the Barratt Impulsiveness Scale version 11 (BIS-11) [20] will be completed at baseline, using the version adapted for use in people with IDD $[21,22]$.

\section{tDCS adverse effects:}

Participants will be monitored for side effects of Active/Sham tDCS treatment using a tDCS Adverse Effects Questionnaire adapted from Brunoni et al [23]. Following each treatment session, participants will be asked to record any side effects related to tDCS.

\section{Participant timeline $\{13\}$}

Figure 1 outlines the proposed study procedure. Eligible participants will have a baseline clinical visit to confirm eligibility criteria and patient capacity, verify severity of aggressive behaviours, and obtain informed consent from participant or designated Substitute Decision Maker. Once enrolled, participants will be randomised into one of two study arms using a block randomization procedure. Participant blinding will be maintained but the research team will be unblinded to study arm.

Participant healthcare records will be examined to obtain information concerning the diagnosis of IDD, demographics, number and duration of admissions to psychiatric institutions and current psychotropic medication regime. A member of staff at the participant's residence with good professional knowledge of 
the participant will be asked to complete the BPI and MOAS at baseline and one week after the last tDCS stimulation. At baseline, participants will be asked to complete the modified BIS-11, and SST. Three tDCS sessions will be delivered over three successive days (one session daily). Participants will be asked to repeat SST at the end of the third session (Figure 1). Additionally, a tDCS adverse effects questionnaire will be administered following each tDCS treatment to detect adverse effects. At one week and also one month following the third and final treatment, participants will complete the BPI and MOAS and will be debriefed to unblind them to treatment arm.

\section{Sample size $\{14\}$}

A power calculation conducted using an independent-samples $t$-test ( $\mathrm{G}^{*} \mathrm{Power}$ ) yielded a total sample size of 60 participants (effect size $=0.65$, power $=80 \%, a=0.05$, one tailed testing).

\section{Recruitment \{15\}}

Participants will be recruited over 24 months from clinics inpatient units and community residential homes for people with IDD.

\section{Assignment of interventions: allocation}

\section{Sequence generation $\{16 \mathrm{a}\}$}

Participants will be randomly assigned to receive either active or sham tDCS using a computer-generated allocation sequence. The research assistant or trial coordinator will be initially blind to the allocation sequence.

\section{Concealment mechanism \{16b\}}

The allocation sequence will be communicated to the research assistant or trial coordinator after completion of baseline measures including BPI, BIS-11 and MOAS.

\section{Implementation $\{16 \mathrm{c}\}$}

A member of the research team other than the principal investigator will generate the allocation sequence. The study's research assistant or coordinator will enroll participants, complete the outcome measures and administer the interventions.

\section{Assignment of interventions: Blinding}

\section{Who will be blinded $\{17 a\}$}

This a single blinded study where participants will be blind to treatment allocation.

\section{Procedure for unblinding if needed $\{17 b\}$}


As the study is single-blinded, the treatment administrator will always know what treatment arm the participants are in, so that in the event of an emergency, administrators can immediately unblind the participant and/or emergency personnel.

\section{Data collection and management}

\section{Plans for assessment and collection of outcomes $\{18 \mathrm{a}\}$}

This information is provided under 'Participant timeline' section of the protocol.

\section{Plans to promote participant retention and complete follow-up $\{18 \mathrm{~b}\}$}

The research team will work closely with participants and their caregivers and Substitute Decision Makers, where applicable, to promote retention and complete follow up. A travel allowance is available to those who are unable to travel to the study administration site.

\section{Data management $\{19\}$}

Refer to the following section of the protocol.

\section{Confidentiality $\{27\}$}

Access to the participants' medical records and study data will be limited to authorized research personnel. Access to electronic data will be password protected and auditable. Electronic data will be stored on a hospital or other institutional network with firewalls and other security and back-up measures in place. Data stored on laptops or mobile devices will be encrypted. Paper copies of study data will be stored in locked filing cabinets in a secure location at Providence Care Hospital in Kingston Ontario. A master linking log with identifiers, accessible only by the principal investigator and research coordinator, will be stored separately from the study data.

\section{Plans for collection, laboratory evaluation and storage of biological specimens for genetic or molecular analysis in this trial/future use $\{33\}$}

Not applicable.

\section{Statistical methods}

\section{Statistical methods for primary and secondary outcomes $\{20 \mathrm{a}\}$}

Behavioural outcome measures will be analyzed using the Statistical Package for Social Sciences (SPSS; Version 26). A $2 \times 2$ Repeated Measures ANOVA will be performed with group allocation (active tDCS vs. sham tDCS) and time (pre- vs. post-tDCS stimulation). Significant main effects and interactions will be explored using simple effects analysis. For instance, within-subject factors will be used to compare changes in outcome variables between the groups over time. A $p$ value of $<0.05$ will be considered as statistically significant. Multiple comparisons will be controlled for with Bonferroni correction. 


\section{Interim analyses $\{21 \mathrm{~b}\}$}

Interim analysis will be conducted once half (30) of the participants have been recruited. Study team members will perform this interim analysis. Stopping rules include: withdrawal of consent, development of a coincidental health problem/illness, exacerbation of aggressive behaviors expressed as a report of a serious violent incident and/or an increased incidence of self-harm or self-injurious behavior. The principal investigator and the Research Ethics Board will make the final decision about early trial termination.

\section{Methods for additional analyses (e.g. subgroup analyses) \{20b\}}

Not applicable.

\section{Methods in analysis to handle protocol non-adherence and any statistical methods to handle missing data $\{20 \mathrm{c}\}$}

Data will be analyzed on an intention-to-treat basis. Multiple imputations technique will be used to deal with missing data.

Plans to give access to the full protocol, participant level-data and statistical code $\{31 \mathrm{c}\}$

Not applicable.

\section{Oversight and monitoring}

\section{Composition of the coordinating centre and trial steering committee $\{5 \mathrm{~d}\}$}

A research management group comprising the trial investigators will be established to manage the overall governance of the project and day-to-day operations. The group will meet monthly to discuss progress and to ensure that the study is conducted in accordance with the requirements of the research ethics approval.

\section{Composition of the data monitoring committee, its role and reporting structure $\{21 \mathrm{a}\}$}

The research management group will be responsible for data monitoring.

\section{Adverse event reporting and harms $\{22\}$}

tDCS is considered to be a safe technique, but some small risks are recognised. A review of 567 tDCS sessions and from questionnaire responses from 102 participants [24] reported that the most common side-effect (reported by $70 \%$ of participants) is that of a tingling sensation under the electrodes. This is present during and shortly after the period of stimulation, and has no adverse effects or risks. Fatigue or tiredness during the stimulation, is the next common report (by about $35 \%$ of participants), and this may continue for a short period afterwards. This may occur when prolonged and uninteresting tasks are used during the experiment. Headaches after stimulation may occur in less than $10 \%$ of the participants. 
Headaches are usually mild and can be treated with normal over-the-counter painkillers, if required. There is no evidence that tDCS leads to any change in frequency or severity of headaches. Overall, less than $20 \%$ of the participants rated the stimulation procedure as mildly unpleasant and $80 \%$ reported that it was not unpleasant. In theory, tDCS might induce seizures, but this has never been reported in the scientific literature.

Potential risks will be completely disclosed to the participants as well as what to do should participants experience any side effects. For instance, should the tingling sensation become painful, the treatment will be stopped. The psychological or emotional risk is minimal; this risk will be mitigated with education and assurance from the study team. Additionally, participants will be debriefed at the end of each session and advised to contact the Principal investigator if they experienced any adverse effects as a result of participation in the study. If a serious adverse event occurs during the treatment sessions, local staff (clinic, hospital, institutional) will be immediately notified and emergency protocols followed. We are not anticipating any adverse events serious or otherwise. However, should one occur, it will be documented and reported to the Research Ethics Board.

\section{Frequency and plans for auditing trial conduct $\{23\}$}

On-site study monitoring will be conducted by designated Providence Care Hospital clinical coordinators who will monitor study safety as serious adverse effects are reported or at least every six months.

\section{Plans for communicating important protocol amendments to relevant parties (e.g. trial participants, ethical committees) $\{25\}$}

Approval to implement important protocol modifications (e.g., changes to eligibility criteria, outcomes, analyses) will be sought from the Queen's University Health Sciences Research Ethics Board (HSREB). Upon receiving approval, the changes will be communicated relevant parties (e.g., investigators, trial participants, and trial registries).

\section{Dissemination plans $\{31$ a $\}$}

The study trial results will be communicated to participants, healthcare professionals, academics and other relevant groups (policy makers) via conference presentations and publication in a peer reviewed journal.

\section{Discussion}

This will be the first randomised controlled trial in the world to assess the efficacy of anodal versus sham tDCS applied to the left DLPFC to reduce impulsivity and aggression in adults with IDD. The proposed study will help elucidate the role of tDCS in reducing impulsivity and incidents of aggression in this population. Assessing the efficacy of repeated tDCS, for instance, delivered daily over a few weeks, and the parameters required to achieve optimal effects will be important considerations in future studies. The results of this study will prompt further research in the field, paving the way for developing targeted 
interventions for modulating impulsivity and aggressive behaviours in people with developmental disabilities.

\section{Trial status}

Protocol version 2 dated July 25, 2020

Date recruitment begins: July 01, 2021

Approximate date when recruitment will be completed: June 30, 2023.

\section{Abbreviations}

\section{BIS-1}

Barratt Impulsiveness Scale version 11

BPI

Behavior Problems Inventory Short Form

DLPFC

Dorsolateral Prefrontal Cortex

IDD

Intellectual developmental disabilities

iTBS

intermittent Theta Burst Stimulation

MOAS

Modified Overt Aggression Scale

RRI

Rapid Response Impulsivity

SSRT

Stop Signal Reaction Time

SST

Stop Signal Task

tDCS

transcranial Direct Current Stimulation

\section{Declarations}

\section{Competing Interests Statement}

All authors have no competing interests.

\section{Acknowledgements}

None. 


\section{Authors' contributions $\{31 \mathrm{~b}\}$}

$\mathrm{NK}$ and ERH developed and wrote the protocol. ERH, AB, JJ, MA contributed to the protocol design and manuscript editing.

\section{Funding $\{4\}$}

This study is funded by the Department of Psychiatry at Queen's University, Canada.

\section{Availability of data and materials $\{29\}$}

The study investigators will have access to the final trial dataset, and there is no contractual agreements that limit such access for investigators. Authorized individuals from Queen's University and Providence Care Hospital will also have access to the final trial to ensure that the study is conducted in accordance with the parameters of ethics approval.

\section{Ethics approval and consent to participate $\{24\}$}

The study has been approved by the Queen's University Health Science \& Affiliated Teaching Hospitals Research Ethics Board, \#PSIY-688-20. All participants or their Substitute Decision Makers will give written informed consent.

\section{Consent for publication $\{32\}$}

Not applicable.

\section{Competing interests $\{28\}$}

This study is funded by the Department of Psychiatry, Queen's University, where the trial investigators are employed. The authors have no other competing interests to declare.

\section{Authors' information (optional)}

Najat Khalifa is Associate Professor in Forensic Psychiatry at the Queen's University Department of Psychiatry.

Emily R Hawken is a behavioural neuroscientist and Assistant Professor in the Psychiatry Department at Queen's University.

Andrew Bickle is an Assistant Professor in Forensic Psychiatry at the Queen's University Department of Psychiatry.

Jessica Jones is a Professor of Psychiatry and Psychology at Queen's University, Department of Psychiatry and a Clinical Forensic Psychologist in the Division of Developmental Disabilities. 
Muhammad Ayub is Professor of Psychiatry at Queen's University, Canada, and University College London, UK.

\section{References}

1. Royal College of Psychiatrists. Challenging Behaviour: A Unified Approach (College Report CR144). 2007. https://www.rcpsych.ac.uk/docs/default-source/improving-care/better-mh-policy/collegereports/college-report-cr144.pdf?sfvrsn=73e437e8_2.

2. Emerson E, Kiernan C, Alborz A, Reeves D, Mason H, Swarbrick R, Mason L, Hatton C. The prevalence of challenging behaviours: a total population study. Res Dev Disabil. 2001. doi:10.1016/s08914222(00)00061-5.

3. Ali A, Blickwedel J, Hassiotis A. Interventions for challenging behaviour in intellectual disability. Adv Psychiatr Treat. 2014. doi:10.1192/apt.bp.113.011577.

4. Drieschner KH, Marrozos I, Regenboog M. Prevalence and risk factors of inpatient aggression by adults with intellectual disabilities and severe challenging behaviour: A long-term prospective study in two Dutch treatment facilities. Res Dev Disabilit. 2013. doi:10.1016/j.ridd.2013.04.008.

5. Cooper SA, Smiley E, Jackson A, Finlayson J, Allan L, Mantry D, Morrison J. Adults with intellectual disabilities: Prevalence, incidence and remission of aggressive behaviour and related factors. $J$ Intellect Disabil Res. 2009. doi:10.1111/j.1365-2788.2008.01127.x.

6. Caswell AJ, Celio MA, Morgan MJ, Duka T. Impulsivity as a multifaceted construct related to excessive drinking among UK students. Alcohol Alcohol. 2016. doi:10.1093/alcalc/agv070.

7. Yang CC, Mauer L, Völlm B, Khalifa N. The use Transcranial Magnetic Stimulation (TMS) and transcranial Direct Current Stimulation (tDCS) to modulate impulsivity in people with mental disorder: A systematic review and explanatory metaanalysis. Neuropsychol Rev. 2020. doi:10.1007/s11065-020-09456-2.

8. Hamilton KR, Littlefield AK, Anastasio NC, Cunningham KA, Fink LH, Wing VC, Mathias CW, Lane SD, Schutz C, Swann AC, Lejuez CW, Clark L, Moeller FG. Potenza, MN. Rapid-Response Impulsivity: Definitions, Measurement Issues, and Clinical Implications. Pers. Disord. 2015. doi:10.1037/per0000100. McGuire J. Assessing Risk of Violence in Offenders with Mental Disorders. In: Beech AR, Craig LA, Chou S, editors. Assessments in Forensic Practice. Browne KD. London: John Wiley \& Sons Ltd; 2017. pp. 137-71.

9. Jolliffe D, Farrington. DP A systematic review of the relationship between childhood impulsiveness and later violence. In: McMurran M, Howard R, editors. Personality, personality disorder and violence: An evidence based approach. London: Wiley-Blackwell; 2009. pp. 41-61.

10. Richard-Devantoy S, Gorwood P, Annweiler C, Olié JP, Le Gall D, Beauchet O. Suicidal behaviours in affective disorders: a deficit of cognitive inhibition? Can J Psychiatry. 2012.

doi:10.1177/070674371205700409. 
11. Bjørkly S. A systematic review of the relationship between impulsivity and violence in persons with psychosis: Evidence or spin cycle? Aggress Violent Behav. 2013. https://doi.org/10.1016/j.avb.2013.08.001.

12. Tracy DK, Shergill SS, David AS, Fonagy P, Zaman R, Downar J, Eliott E, Bhui K. Self-harm and suicidal acts: a suitable case for treatment of impulsivity-driven behaviour with repetitive transcranial magnetic stimulation (rTMS). BJPsych Open. 2015. doi:10.1192/bjpo.bp.115.000315.

13. Emerson C. Challenging Behaviour: Analysis and Intervention in People with Learning Difficulties. Cambridge: Cambridge University Press; 1995.

14. Brevet-Aeby C, Brunelin J, Iceta S, Padovan C, Poulet E. (2016). Prefrontal cortex and impulsivity: interest of noninvasive brain stimulation. NeurosciBiobehav Rev. 2016; doi: 10.1016/j.neubiorev.2016.08.028.

15. Yang CC, Völlm B, Khalifa N. The effects of rTMS on impulsivity in adults: a systematic review and meta-analysis. Neuropsychol Rev. 2018. https://doi.org/10.1007/s11065-018-9376-6.

16. Iwabuchi SJ, Raschke FP, Auer DP, Liddle PF, Lankappa ST, Palaniyappan L. Targeted transcranial theta-burst stimulation alters fronto-insular network and prefrontal GABA. Neurolmage. 2017. doi:10.1016/j.neuroimage.2016.09.043.

17. Neri F, Mencarelli L, Menardi A, Giovannelli F, Rossi S, Sprugnoli G, Rossi A, Pascual-Leone A, Salvador R, Ruffini G, Santarnecchi E. A novel tDCS sham approach based on model-driven controlled shunting. Brain Stimul. 2020. doi:10.1016/j.brs.2019.11.004.

18. Ratey JJ, Gutheil CM. (1991). The measurement of aggressive behavior: Reflections on the use of the Overt Aggression Scale and the modified Overt Aggression Scale. J Neuropsychiatry Clin Neurosci. 1991; 3(2):S57-S60.

19. Rojahn J, Rowe EW, Sharber AC. Hastings RP, Matson JL, Didden R, Kroes DBH, Dumont ELM. (2012). The Behavior Problems Inventory-Short Form (BPI-S) for individuals with intellectual disabilities I: development and provisional clinical reference data. J Intellect Disabil Res. 2012; doi: 10.1111/j.1365-2788.2011.01507.x.

20. Patton JH, Stanford MS, Barratt ES. Factor structure of the Barratt impulsiveness scale. J Clin Psychol. 1995; doi: 10.1002/1097-4679(199511)51:6<768::aid-jclp2270510607>3.0.co;2-1.

21. Parry CJ, Lindsay WR. (2003). Impulsiveness as a factor in sexual offending by people with mild intellectual disability. J Intellectl Disabil Res. 2003; doi: 10.1046/j.1365-2788.2003.00509.x.

22. Snoyman P, Aicken B. (2011). Self-reported impulsivity in male offenders with low cognitive ability in New South Wales prisons. Psychol Crime Law. 2011; https://doi.org/10.1080/10683160903392541.

23. Brunoni AR, Amadera J, Berbel B, Volz MS, Rizzerio BG, Fregni P. (2011). A systematic review on reporting and assessment of adverse effects associated with transcranial direct current stimulation, Int J Neuropsychopharmacol. 2011; https://doi.org/10.1017/S1461145710001690.

24. Poreisz C, Boros K, Antal A, Paulus W. Safety aspects of transcranial direct current stimulation concerning healthy subjects and patients. Brain Res Bull. 2007. doi:10.1016/j.brainresbull.2007.01.004. 


\section{Tables}

Table 1

Inclusion and exclusion criteria

\begin{tabular}{|ll|}
\hline Inclusion criteria & Exclusion criteria \\
\hline $\begin{array}{l}\text { Adults aged 18-64 years } \\
\text { Disanosis of a developmental }\end{array}$ & History of epilepsy or seizures \\
$\begin{array}{l}\text { History of 1 or more incidents of } \\
\text { aggression in the last month }\end{array}$ & $\begin{array}{l}\text { Having metal in the brain/skull, e.g. splinters, fragments or } \\
\text { clips }\end{array}$ \\
$\begin{array}{l}\text { Consent to participate in the trial by the } \\
\text { individual or their Substitute Decision } \\
\text { Maker }\end{array}$ & Having a cochlear implant \\
& $\begin{array}{l}\text { Having an implanted neuro-stimulator (e.g. direct brain } \\
\text { stimulation, epidural/subdural stimulation, vagal nerve } \\
\text { stimulation) }\end{array}$ \\
\hline History of brain surgery of procedure \\
\hline History of severe adverse reaction to tDCS \\
\hline Having a cardiac pacemaker or intracardiac lines \\
\hline Current alcohol or drug misuse \\
\hline Having a sensitive scalp \\
\hline
\end{tabular}

Figures 


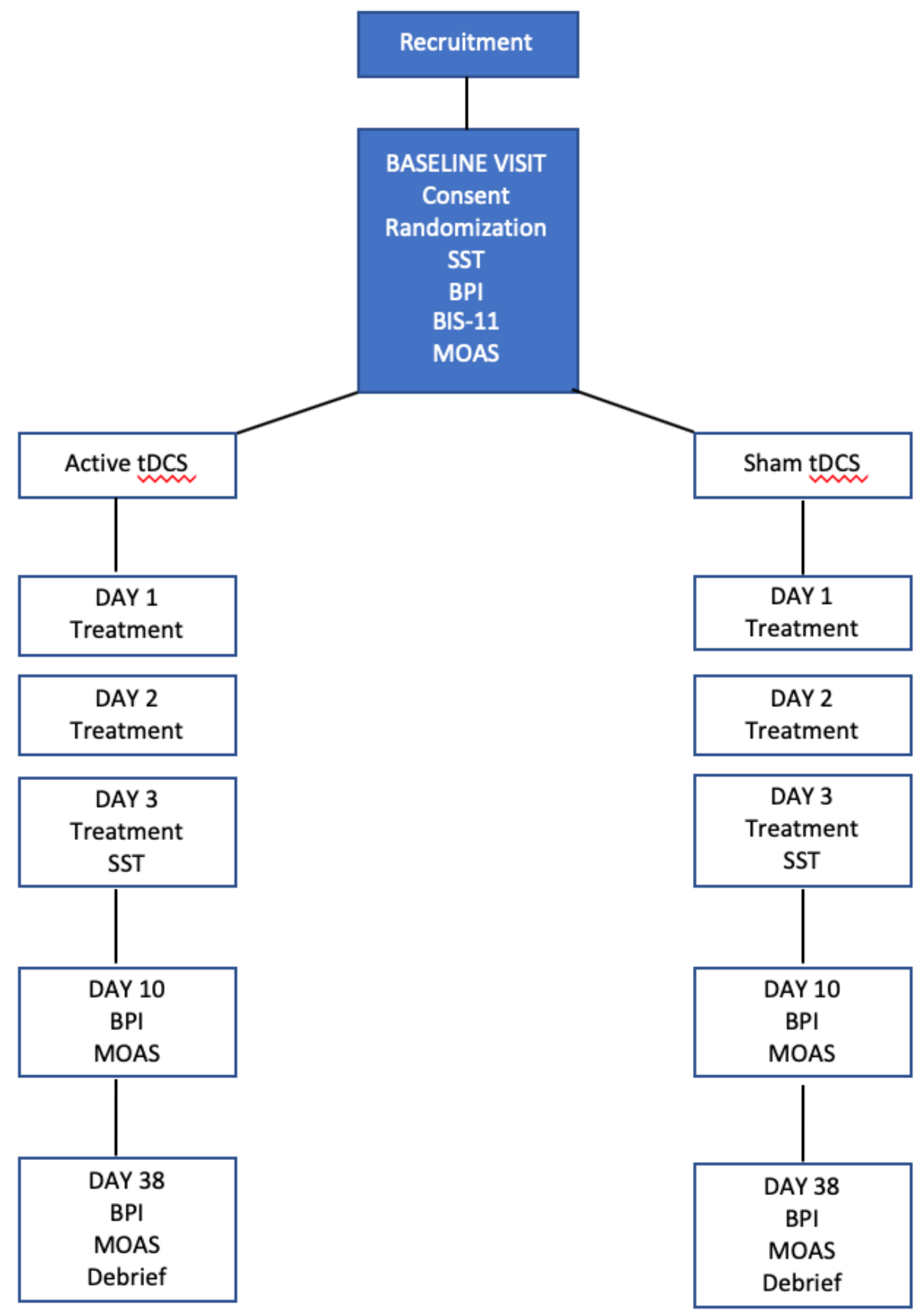

Figure 1

Trial CONSORT diagram Stop signal task (SST), Behavior Problems Inventory (BPI), Barratt Impulsiveness Scale version 11 (BIS-11), Modified Overt Aggression Scale (MOAS) 